\title{
NUMERICAL INTEGRATORS THAT PRESERVE SYMMETRIES AND REVERSING SYMMETRIES*
}

\author{
R. I. MCLACHLAN ${ }^{\dagger}$, G. R. W. QUISPEL ${ }^{\ddagger}$, AND G. S. TURNER ${ }^{\ddagger}$
}

\begin{abstract}
We consider properties of flows, the relationships between them, and whether numerical integrators can be made to preserve these properties. This is done in the context of automorphisms and antiautomorphisms of a certain group generated by maps associated to vector fields. This new framework unifies several known constructions. We also use the concept of "covariance" of a numerical method with respect to a group of coordinate transformations. The main application is to explore the relationship between spatial symmetries, reversing symmetries, and time symmetry of flows and numerical integrators.
\end{abstract}

Key words. numerical integrators, symmetries, automorphisms

AMS subject classifications. 34A50, 70H33, 65L05, 65L06, 65D30, 20D45

PII. S0036142995295807

1. Introduction. Recently there has been a lot of interest in constructing numerical integration schemes for ordinary differential equations (ODEs) in such a way that some qualitative geometrical property of the solution of the ODE is exactly preserved. This has resulted in much work on integration schemes that can preserve the symplectic structure for Hamiltonian ODEs [12, 13, 14, 23, 33]. Other authors have constructed volume-preserving integrators for divergence-free vector fields $[3,18,19,30]$. Yet others again have concentrated on preserving energy and other first integrals $[14,20,21]$ or other mathematical properties [7].

In this paper we are interested in constructing integrators that preserve the symmetries and reversing symmetries of a given ODE. One reason this is important is that nongeneric bifurcations can become generic in the presence of symmetries, and vice versa. One can also consider the time step of the integration scheme as a bifurcation parameter, showing how vitally important it is to stay within the smallest possible class of systems. Reversing symmetries are particularly important, as they give rise to the existence of invariant tori and invariant cylinders [15, 16, 22, 25, 28].

One of the first authors to study integrators that preserve a reversing symmetry was Scovel [24]. His method, as it stands, can only preserve a single reversing symmetry and no ordinary symmetries. In this paper we give a construction of integration schemes preserving an arbitrary number of symmetries and reversing symmetries.

In exploring this question, it became clear that constructions were working because of the effect of certain operators on compositions of maps. This led us to express these effects in terms of automorphisms and antiautomorphisms of groups. In particular, it turns out that many different compositions designed to give methods particular features are all examples of the same general principle. We prepare the basic algebraic material in section 2 .

\footnotetext{
${ }^{*}$ Received by the editors December 6, 1995; accepted for publication (in revised form) December 2, 1996.

http://www.siam.org/journals/sinum/35-2/29580.html

$\dagger$ Mathematics Department, Massey University, Palmerston North, New Zealand (r.mclachlan@ massey.ac.nz).

${ }^{\ddagger}$ Faculty of Science, LaTrobe University, Bundoora, Melbourne 3083, Australia (r.quispel@ latrobe.edu.au). The work of the second author was partially supported by the Australian Research Council.
} 
A curious structure emerges several times. Often a subset of a group is not closed under products $A B$, but is closed under symmetric products $A B A$. We shall call this the sandwich property. It is true for symmetric or antisymmetric matrices, reversible maps, and time-symmetric maps. Subsets with the sandwich property are very useful to us here in integration, although it remains to be seen whether they are an interesting algebraic object in their own right.

Flows of vector fields have many nice properties, and many of these are desirable in an integrator as well. Some of these properties are defined for all vector fields, such as covariance under transformation groups, closure under differentiation [2], and timesymmetry (see (27) below). These are discussed in section 3 . Others are defined only for the flows of a certain class of vector fields, such as symplecticity for Hamiltonian systems, closure under restriction to closed subsystems [2], and energy conservation [14]. We believe that in the context of this new viewpoint of numerical methods, it is natural to try to "lift" these properties so that they are naturally defined for all vector fields. For example, Ge's definition of invariance with respect to symplectic transformations [5] is only defined for Hamiltonian systems, but it can also be seen as a special case of general covariance. In section 4 we study this lift for symmetries and reversing symmetries, and also look at methods that make explicit use of the symmetries of the vector field.

The automorphism point of view elucidates the relationship between different properties, such as time-symmetry and reversibility, which are in fact independent, although related in some special cases considered previously (Runge-Kutta methods with a linear reversing symmetry by Stoffer [27], and splitting into reversible pieces by McLachlan [10]). For example, we show in section 4 how a map covariant under a group larger than strictly necessary can be more flexible under composition.

2. Algebraic preliminaries. Let $G$ be a group with elements $\varphi$ and consider functions $\mathcal{A}: G \rightarrow G$. We only consider functions which are either automorphisms of the group, that is, $\mathcal{A}_{+}$is a bijection and

$$
\mathcal{A}_{+}\left(\varphi_{1} \varphi_{2}\right)=\left(\mathcal{A}_{+} \varphi_{1}\right)\left(\mathcal{A}_{+} \varphi_{2}\right) \quad \forall \varphi_{1}, \varphi_{2} \in G,
$$

or antiautomorphisms, that is, $\mathcal{A}_{-}$is a bijection and

$$
\mathcal{A}_{-}\left(\varphi_{1} \varphi_{2}\right)=\left(\mathcal{A}_{-} \varphi_{2}\right)\left(\mathcal{A}_{-} \varphi_{1}\right) \quad \forall \varphi_{1}, \varphi_{2} \in G .
$$

Examples of automorphisms are the inner automorphisms

$$
\mathcal{N}_{\psi} \varphi:=\psi^{-1} \varphi \psi
$$

where $\psi \in G$. Examples of antiautomorphisms are inverse

$$
\mathcal{I} \varphi:=\varphi^{-1},
$$

and, if $G$ is a linear group (a group of matrices), the transpose $\mathcal{T} \varphi$. In applications to quantum systems, $G$ may be a group of linear operators on a Hilbert space, which has the adjoint antiautomorphism.

Note that the set of automorphisms and antiautomorphisms itself also forms a group, where the group operation is composition. The automorphisms form a normal subgroup. More specifically, every such group $\mathcal{G}_{ \pm}$is homomorphic to $\mathbb{Z}_{2} ;{ }^{1}$ i.e., there is an onto map

$$
\sigma: \mathcal{G}_{ \pm} \rightarrow\{+1,-1\}, \quad \sigma(\mathcal{A}) \sigma(\mathcal{B})=\sigma(\mathcal{A B})
$$

\footnotetext{
${ }^{1}$ Unless there are no antiautomorphisms in the group.
} 
We call the number $\sigma(\mathcal{A})$ the grading of $\mathcal{A}$. In the case at hand, the automorphisms have grading +1 and the antiautomorphisms have grading -1 . Each such group $\mathcal{G}_{ \pm}$ is generated by, for example, its antiautomorphisms alone (if it has any), or by one antiautomorphism together with all the automorphisms.

Define the fixed sets

$$
\operatorname{fix}(\mathcal{A}):=\{\varphi: \mathcal{A} \varphi=\varphi\}
$$

and

$$
\operatorname{fix}(\mathcal{G}):=\{\varphi: \mathcal{A} \varphi=\varphi \forall \mathcal{A} \in \mathcal{G}\} .
$$

Notice that $\operatorname{fix}\left(\mathcal{A}_{+}\right)$is a group,

$$
\varphi_{1}, \varphi_{2} \in \operatorname{fix}\left(\mathcal{A}_{+}\right) \Rightarrow \varphi_{1} \varphi_{2} \in \operatorname{fix}\left(\mathcal{A}_{+}\right),
$$

but fix $\left(\mathcal{A}_{-}\right)$is not. However, we do have that the set of group elements fixed by a given antiautomorphism is closed under the symmetric triple product ("sandwich"):

$$
\varphi_{1}, \varphi_{2} \in \operatorname{fix}\left(\mathcal{A}_{-}\right) \Rightarrow \varphi_{1} \varphi_{2} \varphi_{1} \in \operatorname{fix}\left(\mathcal{A}_{-}\right) .
$$

This concept seems to be useful in, for example, constructing integrators, and we shall call it the sandwich property. ${ }^{2}$

Example. Let $G$ be the linear group $G L(n)$ and consider the antiautomorphism $\mathcal{T}$, transpose. The fixed set of this antiautomorphism is the set of nonsingular symmetric matrices, which has the sandwich property: if $X$ and $Y$ are symmetric matrices, then so is $X Y X$.

Example. Let $G$ be any group and consider the inner automorphism $\mathcal{N}_{\psi}$. The fixed set of this automorphism is the group of so-called $\psi$-equivariant elements: if $\varphi_{1}$, $\varphi_{2}$ are $\psi$-equivariant (i.e., $\varphi_{i}=\psi^{-1} \varphi_{i} \psi$ ), then so is $\varphi_{1} \varphi_{2}$.

If the antiautomorphism $\mathcal{A}_{-}$is an involution (i.e., $\mathcal{A}_{-}^{2}=1$ ) there is a "projection" to $\operatorname{fix}\left(\mathcal{A}_{-}\right)$, namely,

$$
\mathcal{Q}_{\mathcal{A}_{-}} \varphi:=\varphi \mathcal{A}_{-} \varphi,
$$

because $\mathcal{A}_{-}\left(\varphi \mathcal{A}_{-} \varphi\right)=\left(\mathcal{A}_{-}^{2} \varphi\right)\left(\mathcal{A}_{-} \varphi\right)=\varphi \mathcal{A}_{-} \varphi$. $(\mathcal{Q}$ was first introduced in a special case by Benzel, Ge, and Scovel [1].) This can be generalized to a group $\mathcal{G}_{ \pm}$of automorphisms and (not necessarily involutory) antiautomorphisms. Unfortunately, it is difficult to project an arbitrary group element to fix $\left(\mathcal{G}_{ \pm}\right)$; but $\mathcal{Q}_{\mathcal{A}_{-}}$does map the fixed set of the subgroup $\mathcal{G}_{+}$of automorphisms to the fixed set of the entire group.

PROPOSITION 2.1 (generalized Scovel projection). Let $\mathcal{G}_{ \pm}$be a group of automorphisms and antiautomorphisms, and let $\mathcal{G}_{+}$be its subgroup of automorphisms. Let $\mathcal{A}_{-} \in \mathcal{G}_{ \pm}$be an antiautomorphism, so that $\mathcal{G}_{ \pm}=\mathcal{G}_{+} \cup \mathcal{A}_{-} \mathcal{G}_{+}$. Then

$$
\varphi \in \operatorname{fix}\left(\mathcal{G}_{+}\right) \Rightarrow \mathcal{Q}_{\mathcal{A}_{-}} \varphi=\varphi \mathcal{A}_{-} \varphi \in \operatorname{fix}\left(\mathcal{G}_{ \pm}\right) .
$$

(The proof is given below.) This is not a true projection because it does not satisfy $\mathcal{Q}_{\mathcal{A}_{-}}^{2}=\mathcal{Q}_{\mathcal{A}_{-}}$; but it will have some useful properties in our applications.

The "projection" is not always surjective. For example, let $G=G L(n)$ and take a group consisting of one automorphism (the identity) and one antiautomorphism

\footnotetext{
${ }^{2} \mathrm{~A}$ discussion of (what we call) the sandwich property of maps with time-reversal symmetry is given in [9].
} 
TABLE 1 $\left(\begin{array}{cc}0 & I \\ -I & 0\end{array}\right)$.

Sets of matrices as fixed sets of (anti)automorphisms. Here $I$ is the identity matrix and $J=$

\begin{tabular}{|c|c|c|c|c|}
\hline Operator & Sign & Fixed set & Type & "Projection" \\
\hline $\mathcal{T}$ & anti & $A=A^{T}$ & symmetric & $A A^{T}$ \\
$\mathcal{I}$ & anti & $A=A^{-1}$ & involution & $A A^{-1}$ \\
$\mathcal{T} \mathcal{N}_{J}$ & anti & $J A=-(J A)^{T}$ & & $-A J A^{T} J$ \\
\hline $\mathcal{I} \mathcal{T}$ & auto & $A A^{T}=I$ & orthogonal & \\
$\mathcal{I} \mathcal{T} \mathcal{N}_{J}$ & auto & $A J A^{T}=J$ & symplectic & \\
\hline
\end{tabular}

(transpose), that is, $\mathcal{G}_{ \pm}=\{1, \mathcal{T}\} \cong \mathbb{Z}_{2}$. Projection to the symmetric matrices $(A \mapsto$ $A A^{T}$ ) only produces symmetric matrices with nonnegative determinant.

The projection $\mathcal{Q}$ and the sandwich property are in fact both examples of the following more general relationship, which is of central importance in this paper.

PROPOSITION 2.2 (composition property). Let $\mathcal{G}_{ \pm}$be a group of automorphisms $\mathcal{G}_{+}=\left\{\mathcal{A}_{j+}\right\}$ and antiautomorphisms $\mathcal{G}_{-}=\left\{\mathcal{A}_{j-}\right\}$ of the group $G$. Let $\varphi_{1}, \varphi_{2} \in G$ with $\varphi_{1} \in \operatorname{fix}\left(\mathcal{G}_{+}\right)$and $\varphi_{2} \in \operatorname{fix}\left(\mathcal{G}_{ \pm}\right)$. Then

$$
\varphi_{1} \varphi_{2} \mathcal{A}_{j-} \varphi_{1} \in \operatorname{fix}\left(\mathcal{G}_{ \pm}\right)
$$

for all $\mathcal{A}_{j-} \in \mathcal{G}_{-}$.

Proof. We only need to consider the effect of an antiautomorphism; membership of fix $\left(\mathcal{G}_{+}\right)$will follow because the antiautomorphisms generate all of $\mathcal{G}_{ \pm}$:

$$
\begin{aligned}
\mathcal{A}_{k-}\left(\varphi_{1} \varphi_{2} \mathcal{A}_{j-} \varphi_{1}\right) & =\left(\mathcal{A}_{k-}\left(\mathcal{A}_{j-} \varphi_{1}\right)\right)\left(\mathcal{A}_{k-\varphi_{2}}\right)\left(\mathcal{A}_{k-\varphi_{1}}\right) \\
& =\left(\mathcal{A}_{l+} \varphi_{1}\right) \varphi_{2}\left(\left(\mathcal{A}_{j-} \mathcal{A}_{m+}\right) \varphi_{1}\right) \quad \text { for some } l, m \\
& =\varphi_{1} \varphi_{2} \mathcal{A}_{j-} \varphi_{1}
\end{aligned}
$$

for all $k$.

From this proposition the sandwich property follows by taking $\varphi_{1} \in \operatorname{fix}\left(\mathcal{G}_{ \pm}\right)$, and the generalized Scovel projection $\mathcal{Q}$ follows by taking $\varphi_{2}$ to be the identity.

As an example of the composition property $(12)$, take $\mathcal{G}_{ \pm}=\{1, \mathcal{T}\}$. All matrices $B$ are in $\operatorname{fix}\left(\mathcal{G}_{+}\right)$, all symmetric matrices $A$ are in fix $\left(\mathcal{G}_{ \pm}\right)$, and indeed, $B A B^{T}$ is symmetric. The reader is invited to check the consequences of the proposition for the sets of matrices given in Table 1 .

We note that in most of this work, we can weaken the antiautomorphism requirement to

$$
\mathcal{A}_{-}\left(\varphi_{1} \varphi_{2} \varphi_{3}\right)=\left(\mathcal{A}_{-} \varphi_{3}\right)\left(\mathcal{A}_{-} \varphi_{2}\right)\left(\mathcal{A}_{-} \varphi_{1}\right)
$$

for all $\varphi_{1}, \varphi_{2}, \varphi_{3} \in G$. This allows, for example, the operator $\mathcal{A}_{-} A=-A^{T}$, with fixed set the antisymmetric matrices. The composition property (12) is retained, but the projection $(11)$ is lost if the identity is not in $\operatorname{fix}\left(\mathcal{A}_{-}\right)$.

Projecting onto the fixed set of automorphisms is more difficult. However, one may have (as in the case of maps on $\mathbb{R}^{n}$ ) that $G$ is a near-ring: $(G,+,$. ) is a nearring if $(G,+)$ is a group, $(G,$.$) is a semigroup, and (\varphi+\psi) \chi=\varphi \chi+\psi \chi$ (right distributivity). (Note that maps under composition do not form a ring because they are not left-distributive.) If the automorphisms are linear with respect to addition in the near-ring, and $\mathcal{G}_{+}$is a finite group with $\left|\mathcal{G}_{+}\right|$elements, then we can use the 
following, which is a true projection:

$$
\mathcal{P}_{\mathcal{G}_{+}} \varphi=\frac{1}{\left|\mathcal{G}_{+}\right|} \sum_{\mathcal{A}_{+} \in \mathcal{G}_{+}} \mathcal{A}_{+} \varphi
$$

The half-sized group construction. As mentioned above, groups $\mathcal{G}_{ \pm}$are always homomorphic to $\mathbb{Z}_{2}$. In our application to reversing symmetries, we shall use groups $\mathcal{G}_{+}$of automorphisms which themselves are homomorphic to $\mathbb{Z}_{2}$. From such a group we can construct a group $\mathcal{H}_{ \pm}$of the same size as $\mathcal{G}_{+}$in the following way. Let $\mathcal{A}_{+, \sigma}$ denote an element of $\mathcal{G}_{+}$of grading $\sigma= \pm 1$, and let $\mathcal{A}_{-}$be a fixed antiautomorphism whose square $\mathcal{A}_{-}^{2}$ is an element of $\mathcal{G}_{+}$with grading +1 which commutes with $\mathcal{G}_{+}$.

The automorphisms in $\mathcal{H}_{ \pm}$are the elements $\mathcal{A}_{+, 1}$. The antiautomorphisms in $\mathcal{H}_{ \pm}$ are the elements $\mathcal{A}_{-} \mathcal{A}_{+,-1}$. It is straightforward to check that this gives a group $\mathcal{H}_{ \pm}$ of the same size as $\mathcal{G}_{+}$, i.e., half the size of $\mathcal{G}_{ \pm}$.

Example. Let $G$ be $G L(2)$ and $\mathcal{G}_{+}$be the inner automorphisms corresponding to a subgroup of $G$ homomorphic to $\mathbb{Z}_{2}$, say rotations by $\theta=0, \pi / 2, \pi, 3 \pi / 2$. The antiautomorphism $\mathcal{I}$ (inverse) satisfies the above requirements: its square (the identity) is in $\mathcal{G}_{+}$, does have grading +1 , and does commute with $\mathcal{G}_{+}$. The inner automorphisms $\mathcal{N}_{g,+1}$ together with the antiautomorphisms $\mathcal{I N}_{g,-1}$ form the group

$$
\mathcal{H}_{ \pm}=\left\{\mathcal{N}_{0}, \mathcal{N}_{\pi}, \mathcal{I N}_{\pi / 2}, \mathcal{I N}_{3 \pi / 2}\right\}
$$

This group comprises two automorphisms and two antiautomorphisms-it is $\mathbb{Z}_{2} \times \mathbb{Z}_{2}$. Then in order for $\mathcal{Q}_{\mathcal{I N}_{\pi / 2}}$ to be a projection to fix $\left(\mathcal{H}_{ \pm}\right)$we need $\varphi$ to be fixed under all the automorphisms in $\mathcal{H}_{ \pm}$. Here we require $\varphi=\mathcal{N}_{\pi} \varphi$.

3. General properties of integration methods. For simplicity we restrict our attention to systems of first-order autonomous ODEs on $\mathbb{R}^{n}$, i.e., ODEs of the form

$$
\dot{y}=f(y), \quad y \in \mathbb{R}^{n}
$$

for some $n$. Let $F$ be a set of vector fields on $\mathbb{R}^{n}$, and let $\Phi$ be a set of functions ${ }^{3}$

$$
\begin{gathered}
\varphi: F \times[-T, T] \rightarrow \operatorname{Diff}\left(\mathbb{R}^{n}\right), \\
\varphi_{\tau}(f): y \rightarrow \tilde{y} \quad(f \in F, \tau \in[-T, T]) .
\end{gathered}
$$

An element $\varphi \in \Phi$ is called an integrator of $f$ if

$$
\varphi_{\tau}(f): y \mapsto \tilde{y}=y+\tau f(y)+\mathcal{O}\left(\tau^{2}\right) .
$$

When we want to emphasize the functional dependence of $\varphi$ on $\tau$ and $f$ we shall call it a "method"; at other times we may want to think of fixing $\tau$ and $f$ and looking at the resulting map. The allowable time interval $[-T, T]$ may be $\mathbb{R}$, but this will depend on the particular vector fields and maps.

Examples. If all $f \in F$ are differentiable, then Euler's method $\varphi_{\tau}(f)=1+\tau f$, any Runge-Kutta method, and any one-step method which is functionally dependent

\footnotetext{
${ }^{3}$ If $n$ were to depend on $f \in F$, operations which inflate or deflate phase space could be included. But then the group operation (composition of maps) would not be defined for all $\varphi \in \Phi$. More exotic structures-groupoids-would be needed.
} 
only on $f$ are possible members of $\Phi$ (for an appropriate choice of $T$ ). If we impose additional conditions on the set of vector fields $F$, then there is larger family of possible $\varphi$ 's; e.g., if all $f \in F$ have global flows then these flows $\varphi_{\tau}(f)=\exp (\tau f)$ may be in $\Phi$; if all $f \in F$ are $C^{r}$, then the Taylor series method of order $r+1$ may be in $\Phi$. Moreover, functions which are not integrators at all can also be in $\Phi$. Examples are $\varphi_{\tau}(f): y \mapsto y$, and (if all $f$ are $C^{r}$ ), elementary differentials such as $\tau^{2} f^{\prime} f$.

To apply the results of the preceding section, a group is needed. To construct it we let the set of maps

$$
\left\{\varphi_{\tau}(f): \varphi \in \Phi, f \in F, \tau \in \mathbb{R}\right\}
$$

generate a group $G$ by composition. For example,

$$
\varphi_{\tau}(f) \psi_{\sigma}(g) \in G \quad \forall f, g \in F, \tau, \sigma \in \mathbb{R}, \varphi, \psi \in \Phi .
$$

We will not specify the sets $F$ and $\Phi$ precisely, as we wish to make the constructions apply as generally as possible. Minimum requirements will be clear in each application.

Not all the elements of $G$ associate maps with vector fields. For example, let $\varphi_{\tau}(f)$ be an integrator of $f$. Then even though $\psi=\varphi_{\tau}(f) \varphi_{\tau}(g)$ is an integrator of all vector fields $f+g+\mathcal{O}(\tau), \psi$ is not an operator on vector fields. In fact the group $G$ may seem at first to be much bigger than necessary: one could also let, e.g., $\Phi$ generate a group, with group operation

$$
\left(\varphi_{1} \varphi_{2}\right)_{\tau}(f):=\varphi_{1 \tau}(f) \varphi_{2 \tau}(f) .
$$

But we wish to allow flexibility in the choice of time steps (for composition methods) and vector fields (for splitting methods).

The functions $\mathcal{A}$ are now operators on the space of methods. They may or may not preserve the property of being an integrator, and if they do, they may not preserve the order of the integrator.

The exact flows of differential equations are important elements of $G$. It is their properties that we are trying to mimic in constructing actual numerical methods. Some properties (e.g., the semigroup property $\left.\varphi_{\tau}(f) \varphi_{\sigma}(f)=\varphi_{\tau+\sigma}(f)\right)$ hold and are defined for all $f$; others only hold and are defined for some $f$ (e.g., volume-preservation $\operatorname{det}\left(d \varphi_{\tau}(f)\right)=1$ for divergence-free $\left.f\right)$. Where possible, we try to extend the definition of these restricted properties to all $f$; but even with the guiding principle that they should hold for flows, this extension cannot be unique. In this section we consider properties defined for all $f$, in particular, those shared by fixed sets of automorphisms and antiautomorphisms of $G^{4}$

Our first such property is "covariance." It is often useful to consider whether a method produces the same integrator in different coordinate systems. This concept was studied for symplectic transformations by Ge [5] with the aim of preserving integrals of Hamiltonian systems by symplectic integrators. It was also used (with general linear transformations and Runge-Kutta methods) by McLachlan in [11]. We extend this concept to general integrators and arbitrary groups of transformations acting on phase space.

DEFINITION 3.1. Let $H$ be a group with a left action $(h, y) \mapsto$ hy on phase space $\mathbb{R}^{n} \ni y$. A method $\varphi_{\tau}(f)$ is covariant with respect to $H$ if the following diagram

\footnotetext{
${ }^{4}$ Note that if the antiautomorphism $\mathcal{A}_{-}$is such that flows are in $\operatorname{fix}\left(\mathcal{A}_{-}\right)$and $\varphi_{\tau}(f)$ is an integrator of $f$ of a certain order, then so is $\mathcal{A}_{-} \varphi_{\tau}(f)$. This is useful when composing as in (12).
} 
commutes $\forall h \in H$ :

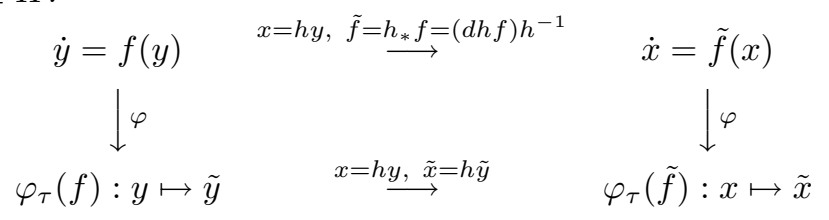

The top arrow pushes forward the vector field $f$ by $h$; the bottom arrow conjugates the map $\varphi_{\tau}(f)$ by $h$. Writing out the transformations through a clockwise loop shows that

$$
\varphi \in \operatorname{fix}\left(\mathcal{K}_{h}\right) \quad \forall h \in H
$$

where

$$
\mathcal{K}_{h} \varphi:=h^{-1} \varphi_{\tau}\left((d h f) h^{-1}\right) h .
$$

(Compare the coordinate transformation automorphism $\mathcal{K}_{h}$ to the inner automorphism $\mathcal{N}_{h} \varphi=h^{-1} \varphi_{\tau}(f) h$, whose fixed set is the equivariant maps.) Now that covariance is defined for $\Phi$ by (24)-(26) (i.e., it is defined for the generators of $G$ ), the definition extends to all of $G$ in the natural way.

If $\varphi_{\tau}(f)$ is the time- $\tau$ flow of $f$, then $\varphi$ is covariant with respect to the group of diffeomorphisms of $\mathbb{R}^{n}$. In general, however, discrete approximations will only be covariant with respect to the group of affine transformations or one of its subgroups. Different methods can then be classified by their covariance group. Many traditional methods, such as Euler's method, the midpoint and trapezoidal rules, and any RungeKutta or Taylor series method, are covariant with respect to the affine group (the semidirect product of $G L(n)$ and the translations $\mathbb{R}^{n}$ ). (Some cases have been treated in $[5,27,31]$.) Most methods are covariant with respect to translations, but some are not covariant with respect to the entire linear group but only with respect to a subgroup. For example, the known volume-preserving methods [3, 19] are covariant with respect to the subgroup of diagonal transformations. Splitting methods which partition the coordinates are covariant with respect to arbitrary transformations which preserve the partitioning.

Our second important property is time-symmetry.

DEFINITION 3.2. A method $\varphi$ is time-symmetric if

$$
\varphi_{\tau}=\varphi_{-\tau}^{-1} .
$$

This important property, also known as "symmetry" or "self-adjointness" or "reversibility," is considered in $[4,6,17,24,26,32,33]$. (We reserve the name "symmetry" for the spatial symmetries considered in the next section.) Because the parameter $\tau$ is included in $G$, we can introduce the time-reversal automorphism

$$
\mathcal{R} \varphi_{\tau}:=\varphi_{-\tau},
$$

so that time-symmetry means being in the fixed set of the antiautomorphism $\mathcal{I R}$. (Recall that $\mathcal{I}=$ inverse.)

(One can also consider inverse-negative $\varphi^{\prime}$ 's with $\varphi_{\tau}(f)=\varphi_{\tau}^{-1}(-f)$ and timescaling $\varphi$ s with $\varphi_{c \tau}(f)=\varphi_{\tau}(f / c)$. However, since there is a simple projection onto the fixed set of the time-scaling operator, namely, $\varphi_{\tau}(f) \mapsto \varphi_{1}(\tau f)$, and the inversenegative and time-symmetry properties are then equivalent, we will not emphasize these properties.) 
We now apply the propositions of section 2 to this group and these (anti)automorphisms. If our favorite integration method is not time-symmetric, we can use the projection

$$
\mathcal{Q}_{\mathcal{I R}} \varphi=\varphi_{\tau} \varphi_{-\tau}^{-1}
$$

to make it so. Now $(\mathcal{I R})^{2}=1$, so the group $\mathcal{G}_{ \pm}$generated by $\mathcal{I} \mathcal{R}$ is just $\{1, \mathcal{I} \mathcal{R}\}$. It has no nontrivial automorphisms so all $\varphi$ are in fix $\left(\mathcal{G}_{+}\right)$and (29) gives a time-symmetric map for any $\varphi$. (This projection was first given in [1].) the form

We can also use the composition property (12) to get time-symmetric methods of

$$
\varphi_{1, \tau} \varphi_{2, \tau} \varphi_{1,-\tau}^{-1},
$$

where $\varphi_{1}$ is any method and $\varphi_{2}$ is time-symmetric, which has been used by many authors starting with Suzuki [29] and Yoshida [33] for constructing higher-order methods.

Now consider the problem of constructing methods which are both covariant and time-symmetric. Take a group of coordinate transformation automorphisms $\mathcal{G}_{+}=$ $\left\{\mathcal{K}_{h}: h \in H\right\}$ and the antiautomorphism $\mathcal{I} \mathcal{R}$ which together generate $\mathcal{G}_{ \pm}^{(1)}$. First, if $\varphi$ is covariant with respect to $H$ (so that $\varphi \in \operatorname{fix}\left(\mathcal{G}_{+}\right)$), then

$$
\varphi \mathcal{I R} \varphi=\varphi_{\tau} \varphi_{-\tau}^{-1} \in \operatorname{fix}\left(\mathcal{I} \mathcal{R} \mathcal{K}_{h}\right)
$$

for all $h \in H$. Second, if $H$, and hence $\mathcal{G}_{+}$, is homomorphic to $\mathbb{Z}_{2}$, then the half-sized group construction gives a subgroup of $\mathcal{G}_{ \pm}^{(1)}$,

$$
\mathcal{G}_{ \pm}^{(2)}=\left\{\mathcal{K}_{h}: h \in H, \sigma(h)=1\right\} \cup\left\{\mathcal{I} \mathcal{R} \mathcal{K}_{g}: g \in H, \sigma(g)=-1\right\}
$$

with projection

$$
\begin{aligned}
\mathcal{Q}_{\mathcal{I R K}_{g}} \varphi & =\varphi \mathcal{I R K}_{g} \varphi \\
& =\varphi_{\tau}(f) g^{-1} \varphi_{-\tau}^{-1}\left((d g f) g^{-1}\right) g
\end{aligned}
$$

where $g$ is an element with grading -1 and $\varphi \in \operatorname{fix}\left(\mathcal{K}_{h}\right)$ for all elements $h$ with grading +1 . All exact flows are in $\operatorname{fix}\left(\mathcal{G}_{ \pm}^{(1)}\right)$ and $\operatorname{fix}\left(\mathcal{G}_{ \pm}^{(2)}\right)$, so these are desirable properties for integrators as well. The advantage of also considering $\mathcal{G}_{ \pm}^{(2)}$ is that then the projection requires less of $\varphi$; it need only be fixed by the +1 -graded elements instead of by all of $H$.

4. Symmetries, reversing symmetries, and integrators. In this section we are interested in properties of integrators that do not hold for all vector fields, but only for those with some given property, such as possessing a symmetry or reversing symmetry. We first give some definitions:

The ODE (17) is $S$-symmetric if the vector field $f$ satisfies

$$
f=(d S f) S^{-1} .
$$

The ODE (17) is $R$-reversible if the vector field $f$ satisfies

$$
f=-(d R f) R^{-1} .
$$

Here $S$ and $R$ are arbitrary diffeomorphisms of phase space; i.e., $R$ is not necessarily an involution. 
The map $\varphi$ is $S$-symmetric if $\varphi \in \operatorname{fix}\left(\mathcal{N}_{S}\right)$, i.e.,

$$
\varphi_{\tau}(f)=S^{-1} \varphi_{\tau}(f) S .
$$

The map $\varphi$ is $R$-reversible if $\varphi \in \operatorname{fix}\left(\mathcal{I N}_{R}\right)$, i.e.,

$$
\varphi_{\tau}(f)=R^{-1} \varphi_{\tau}^{-1}(f) R .
$$

If $f$ is $S$-symmetric, then $S$-symmetry of $\varphi_{\tau}(f)$ is equivalent to $S$-covariance $(\varphi \in$ fix $\left.\left(\mathcal{K}_{S}\right)\right)$. If $f$ is $R$-reversible, then $R$-reversibility of $\varphi_{\tau}(f)$ is equivalent to $\varphi \in$ $\operatorname{fix}\left(\mathcal{I} \mathcal{R} \mathcal{K}_{R}\right)$.

Of course, a dynamical system may possess more than one (reversing) symmetry. The set of all symmetries and reversing symmetries of a given dynamical system form a group under composition, the reversing symmetry group $\Gamma[8,22]$. It is homomorphic to $\mathbb{Z}_{2}$ : the symmetries have grading +1 and the reversing symmetries have grading -1 . The flow $\exp (\tau f)$ of $f$ has the same reversing symmetry group as $f$.

Example. Reversing symmetries originated in classical mechanics, in the following fundamental example. Let $q, p \in \mathbb{R}^{n}$. The Hamiltonian system with Hamiltonian $H=p^{2}+V(q)$, i.e., $\dot{q}=p, \dot{p}=-\nabla V(q)$, has reversing symmetry $(q, p) \mapsto(q,-p)$. If, in addition, $V(q)=V(-q)$, then $(q, p) \mapsto(-q, p)$ is also a reversing symmetry. Their composition, $(q, p) \mapsto(-q,-p)$, is then an ordinary symmetry, giving $\Gamma=\mathbb{Z}_{2} \times \mathbb{Z}_{2}$ in this case.

Example. Ordinary symmetries often arise from a spatial symmetry of physical systems. Consider $H(q, p)=p^{2}+\sum_{i} V\left(\left|q-\alpha_{i}\right|\right)$, i.e., the problem of attraction by fixed centers $\alpha_{i}$. The arrangement of the centers determines the symmetry group. With $n$ centers equally spaced on a circle, it is $D_{n}$, giving $\Gamma=D_{n} \times \mathbb{Z}_{2}$.

Example. Let $L, B(L) \in \mathbb{R}^{n \times n}$ and consider the matrix differential equation $\dot{L}=$ [B, $L]$ with $S: L \mapsto L^{T}$. If $B\left(L^{T}\right)=B^{T}(L)$ (i.e., the map $B$ is $S$-symmetric), then the ODE is $S$-reversible, while if $B\left(L^{T}\right)=-B^{T}(L)$, then the ODE is $S$-symmetric. Similar remarks apply for other matrix (anti)automorphisms in place of $S$.

Constructing integrators possessing a given reversing symmetry group. The question we now address is: given a vector field $f$, possessing a reversing symmetry group $\Gamma$, how does one construct integrators $\varphi_{\tau}(f)$ that possess the same reversing symmetry group? For the case of a single reversing symmetry $R$, Scovel [24] introduced the following projection: if $R^{2}=1$, then $\varphi_{\tau} R \varphi_{\tau}^{-1} R$ is $R$-reversible. This is another instance of the projection (11), in this case, $\mathcal{Q}_{\mathcal{I N}_{R}}$. (Just as for the antiautomorphism $\mathcal{I R}$, the only generated automorphism is the identity.) For the case of a larger reversing symmetry group $\Gamma$, we apply the half-sized group construction. This allows us to use $\varphi$ 's which are not covariant with respect to all of $\Gamma$, but only with respect to its symmetries. Then for any reversing symmetry $R$ in the group, the projection $\mathcal{Q}_{\mathcal{I} \mathcal{N}_{R}} \varphi=\varphi_{\tau} R^{-1} \varphi_{\tau}^{-1} R$ possesses the full reversing symmetry group $\Gamma$.

There are two possible extensions of this projection to all vector fields $f$. The first, $\mathcal{Q}_{\mathcal{I N}_{R}} \varphi$, has the reversing symmetry group $\Gamma$, regardless of whether the vector field does. In particular, it is not an integrator of $f$ even if $\varphi$ is. Thus we prefer the following extension:

$$
\mathcal{Q}_{\mathcal{I R} \mathcal{K}_{R}} \varphi=\varphi_{\tau}(f) R^{-1} \varphi_{-\tau}^{-1}\left((d R f) R^{-1}\right) R .
$$

The conceptual advantage is that all flows are in $\operatorname{fix}\left(\mathcal{I} \mathcal{R} \mathcal{K}_{R}\right)$. This is exactly the group and projection used in (33). 
A drawback of the method $\mathcal{Q}_{\mathcal{I R} \mathcal{K}_{R}} \varphi$ is that it is not time-symmetric, and that making it so by a second projection would destroy the desired reversibility: $\mathcal{Q}_{\mathcal{I} \mathcal{R}}$ $\mathcal{Q}_{\mathcal{I} \mathcal{R} \mathcal{K}_{R}} \varphi \notin \operatorname{fix}\left(\mathcal{I} \mathcal{R} \mathcal{K}_{R}\right)$. There are three ways out.

The first is to use the composition property (12) to increase the order, ignoring time-symmetry completely. For example, if $\varphi_{\tau}$ is reversible and of order 1 , then one can check that

$$
\varphi_{c \tau} \varphi_{(2 c-1) \tau}^{-1} \varphi_{c \tau}, c=1-1 / \sqrt{2}
$$

is reversible and of order 2 .

The second is to argue that time-symmetry does not affect the dynamics of the method as much as reversibility. So we could first construct a nonreversible high-order method $\varphi$ (which may well be time-symmetric), and then form $\mathcal{Q}_{\mathcal{I} \mathcal{R} \mathcal{K}_{R}} \varphi$, preserving the order but not time-symmetry.

The third way is the most interesting. Abstractly, the problem is that we have two antiautomorphisms $\mathcal{A}_{-}$and $\mathcal{B}_{-}$and would like to construct an element invariant under them both. For the projection to work, the initial element $\varphi$ must be invariant under all automorphisms generated by $\mathcal{A}_{-}$and $\mathcal{B}_{-}$. Here we have $\varphi \in \operatorname{fix}\left(\mathcal{A}_{-}^{2}\right)$ and $\varphi \in \operatorname{fix}\left(\mathcal{B}_{-}^{2}\right)$ but not $\varphi \in \operatorname{fix}\left(\mathcal{A}_{-} \mathcal{B}_{-}\right)$. So $\mathcal{Q}_{\mathcal{A}_{-}}$does not map to the fixed set of the full group of anti- and automorphisms. However, if also $\varphi \in \operatorname{fix}\left(\mathcal{A}_{-} \mathcal{B}_{-}\right)$, then it is easy to see that $\varphi$ is fixed by all generated automorphisms, and that projection with any generated antiautomorphism gives the same $\mathcal{Q} \varphi$.

In the case at hand, $\mathcal{A}_{-}=\mathcal{I} \mathcal{R}, \mathcal{B}_{-}=\mathcal{I} \mathcal{R} \mathcal{K}_{R}$, and $\mathcal{A}_{-} \mathcal{B}_{-}=\mathcal{K}_{R}$. Thus we are interested in maps covariant under the reversing symmetry $R$ as well, instead of just the ordinary symmetry $R^{2}$ as previously:

$$
\begin{aligned}
\varphi_{\tau}(f) & =\mathcal{K}_{R} \varphi_{\tau}(f) \\
& =R^{-1} \varphi_{\tau}\left((d R f) R^{-1}\right) R \\
& =R^{-1} \varphi_{-\tau}(f) R \quad \text { if } f \text { is } R \text {-reversible } \\
& =\mathcal{R N}_{R} \varphi_{\tau}(f)
\end{aligned}
$$

Note that if $f$ is $R$-reversible, its flow is $R$-reversible, $R$-covariant, and timesymmetric. If a map satisfies any two of time-symmetry, $R$-reversibility, and $R$ covariance, then it also satisfies the third. This gives a very flexible approach for constructing integrators, for $R$-covariance -invariance under an automorphism-is preserved under arbitrary compositions. At any stage one may project using $\mathcal{Q}_{\mathcal{I R}}$ to gain all three properties.

The case of a reversing symmetry group $\Gamma$ is similar. We would now require full $\Gamma$-covariance: $\varphi=\mathcal{K}_{\mathcal{R}} \varphi=\mathcal{K}_{S} \varphi$ for all symmetries $S$ and reversing symmetries $R$ in the group. (In practice, one only checks covariance under a set of generators of $\Gamma$, such as the reversing symmetries.) For example, affinely covariant methods such as Runge-Kutta are covariant with respect to any affine reversing symmetry group. So by asking a little more of $\varphi_{\tau}(f)$, we can directly apply the symmetric compositions of Yoshida [33] to increase the order while still preserving reversibility.

Thus there are many routes to structure-preserving integrators. The preferred path will depend on what additional structure (e.g., volume-preservation, integrals) the ODE has, and on whether the symmetries and/or the reversing symmetries are linear. First find a first-order method $\varphi_{\tau}(f)$. If it is $\Gamma$-covariant, we are done. If $\Gamma$ is linear, $\mathcal{P}_{\Gamma} \varphi$ (see (15)) is $\Gamma$-covariant, although this may break some structure 
in $\varphi$. (For example, there is no known linearly covariant volume-preserving integrator.) The case when $\varphi$ is covariant only with respect to the symmetries is handled by the generalized Scovel projection, (38). Finally, splitting methods in which the constituent vector fields are not reversible introduce some new possibilities, discussed in the example below.

Example 1 (affine method). One possible way of integrating an ODE whose reversing symmetry group is affine is well known. It is not difficult to show that the implicit midpoint rule

$$
\varphi_{\tau}(f): \quad \tilde{y}=y+\tau f\left(\frac{y+\tilde{y}}{2}\right)
$$

is covariant with respect to the group of affine transformations (as indeed all RungeKutta methods are). It follows that if the vector field $f$ has an affine reversing symmetry group $\Gamma$, then so does $\varphi_{\tau}(f)$. Since the midpoint rule also has time-symmetry $(27), \varphi_{\tau}(f)$ has reversing symmetry group $\Gamma$. (The midpoint rule is also symplectic when the ODE (17) is Hamiltonian [23], but not all Runge-Kutta methods with the time-symmetry property are symplectic, nor vice versa.)

Example 2 (symmetric, volume-preserving splitting). The ABC flow has been widely studied as a model volume-preserving three-dimensional flow. It has phase space $\mathbb{T}^{3}$. We consider the case when two of the parameters are equal, obtaining the "AAC" system:

$$
\begin{aligned}
& \dot{x}=A \sin z+C \cos y, \\
& \dot{y}=A \sin x+A \cos z, \\
& \dot{z}=C \sin y+A \cos x .
\end{aligned}
$$

This system has a reversing symmetry group with 16 elements, and is divergence-free. The reversing symmetry group is generated by the three elements

$$
\begin{aligned}
& R_{1}:(x, y, z) \mapsto(x, \pi-y,-z), \\
& R_{2}:(x, y, z) \mapsto(-x, y, \pi-z), \\
& R_{3}:(x, y, z) \mapsto\left(\frac{3 \pi}{2}+z, \frac{\pi}{2}+y, \frac{3 \pi}{2}-x\right) .
\end{aligned}
$$

A splitting that preserves these properties is

$$
\begin{aligned}
& \dot{x}=A \sin z+C \cos y, \\
& \dot{y}=0, \\
& \dot{z}=C \sin y+A \cos x, \\
& \dot{x}=0, \\
& \dot{y}=A \sin x+A \cos z, \\
& \dot{z}=0 .
\end{aligned}
$$

A second-order, volume-preserving, reversing symmetry group-preserving integrator is therefore

$$
\varphi_{1}(\tau / 2) \varphi_{2}(\tau) \varphi_{1}(\tau / 2)
$$

where $\varphi_{1}$ is the exact solution of (45), and $\varphi_{2}$ is the implicit midpoint rule applied to (44). (Applied directly to (42), a volume-preserving integrator would not in general preserve the symmetries, and a symmetry-preserving integrator, such as the midpoint rule, would not preserve volume.) 
Nonreversible splitting. Because we know how to recover the reversing symmetries, it is not essential that they be preserved in a splitting, as they were in the previous example. Suppose $f=\sum f_{i}$ where each $f_{i}$ possesses all (nonreversing) symmetries (but not necessarily all reversing symmetries) of $f$, and the solution of each $\dot{y}=f_{i}(y)$ can be approximated by a $\varphi_{\tau}\left(f_{i}\right)$ preserving the symmetries and any reversing symmetries which are common to all the $f_{i}$. Then we only need to combine the approximations so as to recover the reversing symmetries. In general, there are three cases.

First, if each $f_{i}$ is reversible, then the $\varphi_{\tau}\left(f_{i}\right)$ are reversible by assumption, so the composition

$$
\varphi_{\tau / 2}\left(f_{1}\right) \ldots \varphi_{\tau / 2}\left(f_{n}\right) \varphi_{\tau / 2}\left(f_{n}\right) \ldots \varphi_{\tau / 2}\left(f_{1}\right)
$$

is reversible - and, if $\varphi_{\tau}(f)$ is time-symmetric, (47) is also time-symmetric.

Second, if there is no structure when the $f_{i}$ are reversed, the best we can do is let

$$
\psi_{\tau}=\varphi_{\tau}\left(f_{1}\right) \ldots \varphi_{\tau}\left(f_{n}\right)
$$

and apply the generalized Scovel projection, giving $\psi_{\tau} R \psi_{\tau}^{-1} R^{-1}$. Time-symmetry of $\varphi$ is irrelevant here.

Third, and perhaps most efficiently, suppose we can split into three pieces, $f_{1}, f_{2}$, and $f_{3}$, with the following structure:

$$
d R\left(f_{1}, f_{2}, f_{3}\right) R^{-1}=-\left(f_{3}, f_{2}, f_{1}\right) .
$$

(The reversible piece $f_{2}$ may be split further if desired.) Let $\varphi_{\tau}\left(f_{2}\right)$ be reversible and $\psi_{\tau}\left(f_{1}\right)$ be any integrator. Then

$$
\psi_{\tau}\left(f_{1}\right) \varphi_{\tau}\left(f_{2}\right) \psi_{-\tau}^{-1}\left(f_{3}\right)
$$

is reversible. This situation occurs frequently when there is a reversing symmetry which is linear and satisfies $R^{2}= \pm 1$. For then let $f_{1}$ be any vector field and define $f_{3}:=-R f_{1} R^{-1}$. Then $f_{1}=-R f_{3} R^{-1}$ and $f_{2}:=f-f_{1}-f_{3}=-R f_{2} R^{-1}$ (because $f$ is reversible by assumption). We only need to find an $f_{1}$ such that this splitting is useful.

Example 3 (nonreversible splitting). Consider the divergence-free vector fields in $\mathbb{R}^{4}$ with parameter $\alpha$

$$
\begin{array}{r}
f=\left(x^{3} z^{2}+\alpha x z^{2} u^{2}\right) \frac{\partial}{\partial x} \\
+\left(-y^{3} u^{2}-\alpha y u^{2} z^{2}\right) \frac{\partial}{\partial y} \\
+\left(x+3 y^{2} u^{2} z\right) \frac{\partial}{\partial z} \\
+\left(-y-3 x^{2} z^{2} u\right) \frac{\partial}{\partial u}
\end{array}
$$

which possess the following reversing symmetry group $\Gamma=\left\{R_{1}, R_{2}, S_{1}, S_{2}\right\}$ :

$$
\begin{aligned}
R_{1}:(x, y, z, u) & \mapsto(y,-x, u,-z), \\
R_{2}:(x, y, z, u) & \mapsto(-y, x,-u, z), \\
S_{1}:(x, y, z, u) & \mapsto(x, y, z, u), \\
S_{2}:(x, y, z, u) & \mapsto(-x,-y,-z,-u)
\end{aligned}
$$


( $\Gamma \cong \mathbb{Z}_{4}$, the cyclic group generated by $R_{1}$ ). We want a method preserving the divergence-free structure, the symmetries, and the reversing symmetries. As in the previous example, the midpoint rule would preserve the reversing symmetry group but is not, in general, volume preserving. It is shown in [17] that the midpoint rule is volume preserving if and only if $P(\lambda)=(-1)^{n} P(-\lambda)$ where $P(\lambda)=\operatorname{det}\left(f^{\prime}-\lambda I\right)$. In $\mathbb{R}^{4}$, this requires the coefficient of $\lambda$ in $P(\lambda)$ to be zero. It can be checked that this coefficient is not zero here.

Instead, we shall preserve volume by using a splitting method. Split $f$ into the planar vector fields

$$
\begin{aligned}
& f_{1}=x^{3} z^{2} \frac{\partial}{\partial x}+\left(-y-3 x^{2} z^{2} u\right) \frac{\partial}{\partial u}, \\
& f_{2}=\alpha x z^{2} u^{2} \frac{\partial}{\partial x}-\alpha y u^{2} z^{2} \frac{\partial}{\partial y}, \\
& f_{3}=-y^{3} u^{2} \frac{\partial}{\partial y}+\left(x+3 y^{2} u^{2} z\right) \frac{\partial}{\partial z},
\end{aligned}
$$

so that each $f_{i}$ is divergence-free and hence Hamiltonian in two dimensions, and has symmetries $S_{1}$ and $S_{2}$ (because their coefficients are odd functions). The $f$ 's also satisfy (49) with $R=R_{i}(i=1,2)$, where the $R_{i}$ are given in (52). We let $\varphi_{\tau}$ be the midpoint rule, which is volume-preserving for these Hamiltonian systems, and apply the third case above (50) to get the reversible method

$$
\varphi_{\tau}\left(f_{1}\right) \varphi_{\tau}\left(f_{2}\right) \varphi_{\tau}\left(f_{3}\right) .
$$

This highlights the point that reversible integrators need not be time-symmetric, nor need the pieces in a splitting method possess the reversing symmetries.

If $\alpha=0$, then $f_{2}=0$, and we have the nice situation of a splitting into two pieces, with the direct composition of their flows (or time-symmetric, $\Gamma$-covariant approximations of them) being reversible. Exactly the same constructions apply to a system with integrals, once basic maps preserving the integrals and symmetries have been found.

\section{REFERENCES}

[1] S. Benzel, Z. Ge, And C. Scovel, Elementary construction of higher order Lie-Poisson integrators, Phys. Lett. A, 174 (1993), pp. 229-232.

[2] P. Bochev And C. Scovel, On quadratic invariants and symplectic structure, BIT, 34 (1994), pp. 337-345.

[3] K. FENG AND D.-L. WANG, Dynamical systems and geometric construction of algorithms, in Computational Mathematics in China, Contemp. Math. 163, Z. Shi and C. Yang, eds., AMS, Providence, RI, 1994.

[4] É. Forest, J. Bengtsson, And M. F. Reusch, Application of the Yoshida-Ruth techniques to implicit integration and multi-map explicit integration, Phys. Lett. A, 158 (1991), pp. 99101.

[5] Z. GE, Equivariant symplectic difference schemes and generating functions, Phys. D, 49 (1991), pp. 376-386.

[6] E. Hairer, S. P. Nørsett, and G. Wanner, Solving Ordinary Differential Equations I, Springer-Verlag, New York, 1993.

[7] P. E. Kloeden and K. J. Palmer, eds., Chaotic Numerics, Contemp. Math. 172, AMS, Providence, RI, 1994.

[8] J. S. W. Lamb, Reversing symmetries in dynamical systems, J. Phys. A, 25 (1992), pp. 925-937.

[9] J. S. W. LAMB AND H. BRAnds, Symmetries and reversing symmetries in kicked systems, in Dynamics, Bifurcations and Symmetries, P. Chossat, ed., Kluwer, Dordrecht, the Netherlands, 1994, pp. 181-196. 
[10] R. I. MCLachlan, On the numerical integration of ordinary differential equations by symmetric composition methods, SIAM J. Sci. Comput., 16 (1995), pp. 151-168.

[11] R. I. MCLachlan, Comment on "Poisson schemes for Hamiltonian systems on Poisson manifolds," Comp. Math. Appl., 29 (1995), p. 1.

[12] R. I. McLachlan and P. Atela, The accuracy of symplectic integrators, Nonlinearity, 5 (1992), pp. 541-562.

[13] R. I. McLachlan And C. Scovel, A survey of open problems in symplectic integration, in Integration Algorithms and Classical Mechanics, J. E. Marsden, G. W. Patrick, and W. F. Shadwick, eds., AMS, Providence, RI, 1996, pp. 151-180.

[14] J. E. Marsden, Lectures in Mechanics, Cambridge University Press, Cambridge, UK, 1992.

[15] J. Moser, Convergent series expansions for quasi-periodic motions, Math. Ann., 169 (1967), pp. 136-176.

[16] A. Politi, G. L. Oppo, And R. BAdil, Coexistence of conservative and dissipative behavior in reversible dynamical systems, Phys. Rev. A, 33 (1986), pp. 4055-4060.

[17] M.-Z. QIN AND W.-J. ZHU, Construction of higher order symplectic schemes by composition, Computing, 47 (1992), pp. 309-321.

[18] M.-Z. QIN AND W.-J. ZHU, Volume-preserving schemes and numerical experiments, Comput. Math. Appl., 26 (1993), pp. 33-42.

[19] G. R. W. QuisPel, Volume-preserving integrators, Phys. Lett. A, 206 (1995), pp. 26-30.

[20] G. R. W. Quispel AND H. W. CAPEL, Solving ODE's numerically while preserving a first integral, Phys. Lett. A, 218 (1996), pp. 223-228.

[21] G. R. W. Quispel And H. W. CAPEL, Solving ODE's numerically while preserving all first integrals, submitted.

[22] J. A. G. Roberts And G. R. W. Quispel, Chaos and time-reversal symmetry: Order and chaos in reversible dynamical systems, Phys. Rep., 216 (1992), pp. 63-177.

[23] M. Sanz-Serna And M. P. Calvo, Numerical Hamiltonian Problems, Chapman and Hall, London, 1994.

[24] J. C. Scovel, Symplectic numerical integration of Hamiltonian systems, in The Geometry of Hamiltonian Systems, Tudor Ratiu, ed., MSRI Publ. 22, Springer-Verlag, New York, 1991, pp. 463-496.

[25] M. B. Sevryuk, Reversible systems, Lecture Notes in Mathematics 1211, Springer-Verlag, Berlin, 1986.

[26] H. J. STETTER, Symmetric two-step algorithms for ordinary differential equations, Computing, 5 (1970), pp. 267-280.

[27] D. Stoffer, Variable steps for reversible integration methods, Computing, 55 (1995), pp. 1-22.

[28] A. Stuart, Numerical analysis of dynamical systems, in Acta Numerica, A. Iserles, ed., Cambridge University Press, Cambridge, UK, 1994, pp. 467-572.

[29] M. SuZUKI, Fractal decomposition of exponential operators with applications to many-body theories and Monte-Carlo simulations, Phys. Lett. A, 146 (1990), pp. 319-323.

[30] A. Thyagaraja And F. A. HaAs, Representation of volume-preserving maps induced by solenoidal vector fields, Phys. Fluids, 28 (1985), pp. 1005-1007.

[31] G. Turner, Three-Dimensional Reversible Mappings, Ph.D. thesis, La Trobe University, Victoria, Australia, 1994.

[32] G. WANNER, Runge-Kutta methods with expansions in even powers of $h$, Computing, 11 (1973), pp. 81-85.

[33] H. Yoshida, Construction of higher order symplectic integrators, Phys. Lett. A, 150 (1990), pp. 262-268. 